\title{
Variabilidade espacial das frações da matéria orgânica do solo em área degradada sob recuperação
}

Luiz F. C. Leite ${ }^{1}$, Juscélia da S. Ferreira ${ }^{2}$, Marcos E. da C. Veloso ${ }^{3}$, Francisco E. P. Mousinho ${ }^{4} \&$ Agenor F. Rocha Junior ${ }^{5}$

\section{Palavras-chave:}

carbono orgânico do solo substâncias húmicas geoestatística krigagem

\begin{abstract}
R E S U M O
Objetivou-se, neste estudo, avaliar a variabilidade espacial das frações da matéria orgânica do solo em uma área degradada em recuperação com pinhão-manso; para isto se estabeleceu, em um Argissolo Vermelho-Amarelo, uma malha de 30 por $30 \mathrm{~m}$ para amostragem, totalizando 55 pontos. Em cada ponto foram coletadas amostras de solo nas camadas de $0-0,05$; 0,05-0,10 e 0,10-0,20 m e determinados carbono orgânico total (COT), carbono lábil (CL) e o carbono das frações ácido fúlvico (C-FAF), ácido húmico (C-FAH) e humina (C-HUM). Os maiores teores de COT foram observados na camada de $0-0,05 \mathrm{~m}$ e variaram de 2,0 a 15,5 $\mathrm{g} \mathrm{kg}^{-1}$. O C-FAF nas camadas de 0-0,05 e 0,10-0,20 m e o C-FAH na camada de 0,10-0,20 m apresentaram altos coeficientes de variação (CV) de 66, 74 e 77\%, respectivamente. As demais variáveis apresentaram $\mathrm{CV}$ médio, variando de 32 a $58 \%$. Com exceção do C-FAF e C-HUM (0,0-0,05 m) os demais atributos apresentaram dependência espacial classificada como moderada ou forte, com alcances variando de 39,60 a 410,90 m. Os baixos valores observados para o carbono orgânico total e suas frações realçam o estado de degradação do solo indicando a necessidade de aumento na entrada de carbono por meio da inclusão de espécies com elevado aporte de resíduos.
\end{abstract}

\section{Key words:}

soil organic carbon

humic substances

geostatistics

kriging

\section{Spatial variability of the soil organic matter fractions in degraded area under recovery}

\begin{abstract}
A B S T R A C T
The present study aimed to evaluate the spatial variability of soil organic matter fractions in degraded area under recovery by planting Jatropha curcas. For this purpose, a grid of 30 by $30 \mathrm{~m}$ was established for sampling, totalizing 55 points. At each point, soil samples were collected in the $0-0.05,0.05-0.10$ and $0.10-0.20 \mathrm{~m}$ depths. Total organic carbon (TOC), labile carbon (LC) and fulvic acid fraction (FAF), humic acid fraction (HAF) and humin fraction (HUM) carbon were determined. The FAF at 0-0.05 and 0.10-0.20 m depths and FAH at $0.10-0.20 \mathrm{~m}$ depth showed coefficient of variation of 66,74 and $77 \%$, respectively, considered high. The other variables showed mean CV ranging from 32 to $58 \%$. With the exception of the FAF and HUM (0-0.05 m), the other attributes showed spatial dependence classified as moderate or strong, varying from 39.6 and $410.9 \mathrm{~m}$. Very low values of the total organic carbon and their fractions highlighted the soil degradation and indicate the need to increase carbon inputs using crops with high amount of residues.
\end{abstract}

Protocolo 072-2014 - 07/03/2014 • Aprovado em 14/11/2014 • Publicado em 02/03/2015

${ }^{1}$ Embrapa Emio-Norte. Teresina, PI. E-mail: luiz.f.leite@embrapa.br (Autor correspondente)

${ }^{2}$ UFRPE. Recife, PE. E-mail: jusce_17@yahoo.com.br

${ }^{3}$ Embrapa Meio-Norte. Teresina, PI. E-mail: marcos.emanuel@embrapa.br

${ }^{4}$ Colégio Técnico de Teresina. Teresina, PI. E-mail: edinaldomousinho@yahoo.com.br

${ }^{5}$ Embrapa Meio-Norte. Teresina, PI. E-mail: agenorrochabsbpi@hotmail.com. Bolsista DTI 3 


\section{INTRODUÇÃO}

A degradação do solo é definida como um processo que reduz e/ou elimina a capacidade atual ou potencial do solo para produzir bens ou serviços tornando-se, assim, uma ameaça aos ciclos hidrológicos e biogeoquímicos, à biodiversidade e à produtividade vegetal primária (Marchetti et al., 2012).

Os mecanismos associados à degradação dos solos podem ser evidenciados por meio de atributos físicos, tais como o declínio na agregação do solo, com consequente aumento da erosão e compactação do solo, atributos químicos, tais como a acidificação, salinização e esgotamento da fertilidade do solo e frações químicas da matéria orgânica e atributos biológicos no que tange à diminuição do aporte de carbono e da atividade e diversidade microbiana (Moebius-Clune et al., 2011). Este último tem relação direta com problemas associados à eutrofização das águas superficiais, à contaminação das águas subterrâneas e às emissões de gases $\left(\mathrm{CO}_{2}, \mathrm{CH}_{4}, \mathrm{~N}_{2} \mathrm{O}, \mathrm{NOx}\right)$ de ecossistemas terrestres e aquáticos para a atmosfera. Além desses aspectos biofísicos a degradação do solo é um processo impulsionado também por fatores socioeconômicos e políticos (Imoke et al., 2010).

No sudoeste do Piauí, o município de Gilbués, considerado núcleo de desertificação do nordeste, apresenta comprometimento da economia e meio ambiente devido essencialmente à degradação do solo. A extensão e a intensidade da degradação são elevadas, sobretudo ao se atravessar a região no período seco quando a erosão eólica predomina na mobilização do material emprestando, à paisagem, fisionomia semelhante à de desertos. Isto tem contribuído para a alteração microclimática afetando a biodiversidade e expandindo a desertificação (Sales, 2003).

Nessas áreas, com elevado grau de antropização, o equilíbrio dinâmico é quebrado e as entradas de carbono passam a ser menores que as saídas, o que conduz à redução da quantidade e modificação da qualidade da matéria orgânica do solo (MOS) (Leite et al., 2010). Tais perdas de carbono estão diretamente associadas à diminuição no aporte de material orgânico, ao aumento da taxa de decomposição dos resíduos vegetais e à diminuição da proteção física da MOS (Behesthi et al., 2012).

A MOS é heterogênea e consiste de diferentes frações funcionais e biológicas variando dinâmica e quimicamente. Essas frações respondem de forma diferenciada ao manejo do solo e as mudanças no uso da terra e podem ser classificadas em lábeis e estáveis (Kelleher et al., 2006). A fração lábil apresenta alta taxa de decomposição e um curto período de permanência no solo (meses ou alguns anos) e é considerada importante fonte de C (von Lützow et al., 2007). Por outro lado, a fração estável ou humificada, que representa cerca de $2 / 3$ do C orgânico em áreas tropicais, tem um tempo de permanência no solo que pode chegar a centenas de anos e sua principal função é atuar sobre as condições físicas e químicas do solo além de ser importante dreno de C atmosférico (Stevenson, 1994;
Figueiredo et al., 2010). As frações da MOS, especialmente as lábeis, respondem mais rapidamente às interferências antrópicas quando comparadas com os demais atributos do solo ou mesmo com o carbono orgânico total, o que as torna excelentes indicadores de qualidade do solo (Salvo et al., 2010).

Para se conhecer a variação espacial da dinâmica da MOS e de suas frações, essenciais para a elaboração de estratégias de manejo que promovam o uso sustentável do solo, tem-se utilizado sistematicamente a geoestatística, considerada ferramenta útil para análise e caracterização dos dados espacialmente relacionados (Chaves \& Farias, 2008). A associação das frações da MOS e das características do local pode auxiliar na formulação e na avaliação de modelos baseados em processos e na determinação dos efeitos do uso da terra e da mudança climática nos estoques de carbono (Zong-Ming et al., 2010).

São escassos, na literatura, os estudos sobre a distribuição espacial das frações da MOS (Marchetti et al., 2012) em particular em áreas sob processo de degradação. Neste sentido, o presente estudo objetivou avaliar a variabilidade espacial das frações da matéria orgânica do solo em uma área degradada em recuperação com pinhão-manso, localizado em Gilbués, sudoeste do Piauí.

\section{Material e Métodos}

Este trabalho foi desenvolvido no município de Gilbués (09 51' 18” S, 5 22' 05” W, $481 \mathrm{~m}$ ), sudoeste do estado do Piauí, considerado um dos quatro núcleos de desertificação do nordeste (Brasil, 1998). De acordo com a classificação de Koppen, o clima da região é do tipo Aw (Clima Tropical Chuvoso), com temperatura média de $26,5^{\circ} \mathrm{C}$ e precipitação anual de $1200 \mathrm{~mm}$, com estação chuvosa nos meses de outubro a abril. O solo foi classificado como Argissolo VermelhoAmarelo (EMBRAPA, 2006), e com altos teores de areia e silte o que, associado às elevadas intensidades eventuais de precipitações pluviais, o torna muito vulnerável à erosão hídrica. As características químicas e físicas do solo estão na Tabela 1.

A área de estudo esteve sob floresta nativa de Cerrado até 1940. A partir de sua remoção iniciou-se um processo desordenado de mineração que, associado ao uso desordenado do fogo e pastoreio, contribuiu para aumentar a exposição do solo com remoção total dos horizontes superficiais e exposição, em especial o horizonte $\mathrm{C}$, causando o aparecimento de processos erosivos e a consolidação de um estado de degradação. Em novembro de 2009 iniciou-se a recuperação da área que possui relevo ondulado (declividade média de $4 \%$ ), com o uso de terraços e a revegetação e implantação da cultura do pinhão-manso (Jatropha curcas L.) utilizando-se o espaçamento $3 \times 4 \mathrm{~m}$ (833 plantas ha ${ }^{-1}$ ). A adubação, por ocasião do plantio, foi de $40 \mathrm{~kg}$ de $\mathrm{N} \mathrm{ha}^{-1}, 70 \mathrm{~kg}$ de $\mathrm{P}_{2} \mathrm{O}_{5} \mathrm{ha}^{-1}$ e $20 \mathrm{~kg}$ de $\mathrm{K}_{2} \mathrm{O} \mathrm{ha} \mathrm{h}^{-1}$.

Tabela 1. Caracterização química e física do solo da área experimental nas camadas de 0-0,20 e 0,20-0,40 m

\begin{tabular}{|c|c|c|c|c|c|c|c|c|c|c|c|c|}
\hline \multirow{2}{*}{$\begin{array}{c}\text { Camadas } \\
\text { (cm) }\end{array}$} & \multirow{2}{*}{$\begin{array}{c}\mathrm{pH} \\
\mathrm{H}_{2} \mathrm{O}\end{array}$} & \multirow{2}{*}{$\begin{array}{c}\mathrm{MO} \\
\mathrm{g} \mathrm{kg}^{-1}\end{array}$} & $P$ & $\mathrm{~K}$ & $\mathrm{Ca}$ & $\mathrm{Mg}$ & $\mathrm{Na}$ & $\mathrm{Al}$ & $\mathrm{H}+\mathrm{Al}$ & Areia & Silte & Argila \\
\hline & & & \multicolumn{7}{|c|}{$m g d^{-3}$} & \multicolumn{3}{|c|}{$\mathrm{g} \mathrm{kg}^{-1}$} \\
\hline $0,0-0,20$ & 7,67 & 6,91 & 131,20 & 0,25 & 30,14 & 3,52 & 0,19 & 0 & 0 & 519,50 & 389,50 & 91,00 \\
\hline $0,20-0,40$ & 8,07 & 6,43 & 39,65 & 0,14 & 31,44 & 2,84 & 0,36 & 0 & 0 & 614,40 & 307,30 & 78,50 \\
\hline
\end{tabular}


Em novembro de 2010 definiu-se uma malha de 30 por $30 \mathrm{~m}$ de distância entre um ponto de amostragem e outro, totalizando 55 pontos; em cada ponto foram coletadas três amostras de solo simples, nas camadas de 0-0,05; 0,05-0,10 e 0,10-0,20 m, totalizando 165 amostras as quais foram acondicionadas em sacos plásticos, identificados e encaminhados ao laboratório no qual foram destorroadas, secadas ao ar e passadas em peneiras de $2 \mathrm{~mm}$.

O carbono orgânico total do solo (COT) foi determinado por oxidação via úmida utilizando-se solução de dicromato de potássio em meio ácido, com aquecimento externo (Yeomans \& Bremner, 1988). O carbono orgânico lábil $\left(C_{L}\right)$ foi quantificado por meio de oxidação com $\mathrm{KMnO}_{4}\left(33 \mathrm{mmol} \mathrm{L}{ }^{-1}\right)$, como proposto por Blair et al. (1995) e modificado por Shang \& Tiessen (1997). O carbono não-lábil $\left(C_{N L}\right)$, equivalente ao $\mathrm{C}$ não oxidado pelo $\mathrm{KMnO}_{4}$, foi determinado por diferença $\left(\mathrm{C}_{\mathrm{NL}}=\mathrm{COT}-\mathrm{C}_{\mathrm{L}}\right)$. As substâncias húmicas: carbono da fração ácidos fúlvicos (C-FAF) carbono da fração ácidos húmicos (C-FAH) e carbono da fração huminas (C-HUM) foram obtidas pelo fracionamento químico da MOS com base no método Kononova-Belchikova que se baseia na extração da matéria orgânica com pirofosfato de sódio e hidróxido de sódio. $\left(\mathrm{NaOH} 0,01 \mathrm{~mol} \mathrm{~L}^{-1}+\mathrm{Na}_{4} \mathrm{P}_{2} \mathrm{O}_{7} 0,01 \mathrm{~mol} \mathrm{~L}^{-1}\right)$ (Kononova, 1982).

Realizou-se a análise descritiva e exploratória dos dados com o intuito de confirmar seu ajuste na distribuição normal e para verificar a presença de valores extremos, candidatos a outliers. O grau de variabilidade das variáveis na área de estudo foi classificado por meio do coeficiente de variação (CV\%) de acordo com a classificação proposta por Warrick $\&$ Nielsen (1980), em que considera variabilidade baixa para CV menor que $12 \%$, média para CV entre 12 e $62 \%$ e alta para CV maior $62 \%$.

Confirmada a normalidade dos dados, a inexistência de valores extremos e as condições de estacionaridade, o semivariograma foi gerado considerando-se isotropia dos dados e se utilizando o software GS+. O ajuste do modelo de semivariograma escalonado possibilitou definir os seguintes parâmetros: efeito pepita $(\mathrm{C} 0)$, patamar $(\mathrm{C} 0+\mathrm{C})$, alcance (a) e o índice de dependência espacial (IDE). O IDE foi calculado pela relação $[\mathrm{C} /(\mathrm{C} 0+\mathrm{C})]^{*} 100$, sendo o grau de dependência espacial das variáveis obtido de acordo com a classificação proposta por Cambardella et al. (1994), que considera dependência espacial forte quando o valor do efeito pepita for menor ou igual a $25 \%$ do patamar, moderada para valores entre 25 e $75 \%$ e fraca quando for maior que $75 \%$.

Utilizando os parâmetros do semivariograma, foram gerados os mapas temáticos de COT, CL, C-FAF, C-FAH e C-HUM para cada profundidade estudada utilizando-se para interpolação dos dados o estimador espacial denominado krigagem, método de inferência espacial, o qual estima dados em pontos não amostrados a partir de pontos amostrados com base na estrutura de dependência espacial do fenômeno (Soares, 2006).

\section{Resultados e Discussão}

Os maiores teores de COT foram observados na camada de 0-0,05 m e variaram de 2,0 a 15,5 $\mathrm{g} \mathrm{kg}^{-1}$, com uma média de $7,8 \mathrm{~g} \mathrm{~kg}^{-1}$, considerada muito baixa (Tabela 2). Matias et al. (2009) também observaram, em Latossolo Amarelo distrófico do sudoeste piauiense, em áreas sob sistema de plantio convencional e direto, teores de COT de 20,3 e 28,3 g $\mathrm{kg}^{-1}$, respectivamente, na camada de 0,0-0,05 m, significando 61 e $72 \%$ maiores que o observado neste estudo. Os valores de média e mediana $\left(7,2 \mathrm{~g} \mathrm{~kg}^{-1}\right)$ para o COT e demais variáveis foram considerados próximos o que caracteriza distribuição simétrica (Tabela 2).

Nas camadas de 0-0,05 e 0,05-0,10 m, os teores de CL variaram de 0,1 a $1,0 \mathrm{~g} \mathrm{~kg}^{-1}$ e na camada de 0,10-0,20 m, de 0,1 a $0,9 \mathrm{~g} \mathrm{~kg}^{-1}$ (Tabela 2). Isto representa apenas 10 e $6 \%$ do COT, para as primeiras duas camadas e $6 \%$ para a última, abaixo de outros resultados de CL/COT reportados na literatura

Tabela 2. Análise estatística descritiva dos teores de carbono orgânico total (COT), carbono lábil (CL), carbono não lábil (CNL), carbono da fração ácido fúlvico (C-FAF), ácido húmico (C-FAH), e fração humina (C-HUM), nas camadas de 0-0,05; 0,05-0,10; e 0,10-0,20 m de um Argissolo Vermelho-Amarelo, em Gilbués-Piauí

\begin{tabular}{|c|c|c|c|c|c|c|c|c|}
\hline \multirow{2}{*}{ Estatística } & Média & Mediana & Mínimo & Máximo & Variância & Assimetria & Curtose & \multirow{2}{*}{$\begin{array}{l}\text { CV } \\
\text { (\%) }\end{array}$} \\
\hline & \multicolumn{7}{|c|}{$\left(\mathrm{g} \mathrm{kg}^{-1}\right)$} & \\
\hline & \multicolumn{8}{|c|}{$0-0,05 \mathrm{~m}$} \\
\hline COT & 7,8 & 7,2 & 2,0 & 15,5 & 8,9 & 0,8 & 0,7 & 38,0 \\
\hline $\mathrm{CL}$ & 0,5 & 0,5 & 0,1 & 1,0 & 0,0 & 0,0 & 0,6 & 32,8 \\
\hline CNL & 7,3 & 6,7 & 1,8 & 14,9 & 8,6 & 0,8 & 0,7 & 40,1 \\
\hline C-FAF & 1,0 & 0,8 & 0,1 & 3,3 & 0,5 & 1,4 & 2,4 & 66,6 \\
\hline C-FAH & 2,1 & 1,8 & 0,1 & 5,7 & 1,2 & 0,8 & 1,4 & 51,7 \\
\hline \multirow[t]{2}{*}{$\mathrm{C}-\mathrm{HUM}$} & 4,7 & 4,6 & 0,6 & 13,5 & 7,8 & 1,1 & 1,2 & 58,8 \\
\hline & \multicolumn{8}{|c|}{$0,05-0,10 \mathrm{~m}$} \\
\hline COT & 8,1 & 7,7 & 2,4 & 15,2 & 11,2 & 0,4 & $-0,6$ & 41,3 \\
\hline $\mathrm{CL}$ & 0,5 & 0,5 & 0,1 & 1,0 & 0,0 & $-0,1$ & 0,3 & 33,4 \\
\hline CNL & 7,6 & 7,1 & 2,3 & 14,6 & 10,7 & 0,5 & $-0,6$ & 43,3 \\
\hline C-FAF & 0,8 & 0,8 & 0,1 & 2,3 & 0,2 & 1,0 & 1,6 & 58,9 \\
\hline C-FAH & 1,7 & 1,7 & 0,0 & 4,5 & 0,9 & 0,6 & 0,6 & 54,8 \\
\hline \multirow[t]{2}{*}{$\mathrm{C}-\mathrm{HUM}$} & 5,5 & 4,6 & 0,5 & 12,8 & 9,7 & 0,6 & $-0,6$ & 56,2 \\
\hline & \multicolumn{8}{|c|}{$0,10-0,20 \mathrm{~m}$} \\
\hline COT & 7,0 & 6,3 & 1,5 & 14,9 & 11,6 & 0,6 & $-0,3$ & 48,7 \\
\hline $\mathrm{CL}$ & 0,4 & 0,4 & 0,1 & 0,9 & 0,0 & 0,4 & 0,9 & 38,4 \\
\hline CNL & 6,6 & 5,6 & 0,6 & 14,4 & 11,5 & 0,6 & $-0,2$ & 51,6 \\
\hline C-FAF & 0,5 & 0,5 & 0,0 & 1,6 & 0,2 & 0,7 & $-0,1$ & 77,1 \\
\hline C-FAH & 1,1 & 1,1 & 0,0 & 3,3 & 0,7 & 0,7 & $-0,1$ & 74,9 \\
\hline $\mathrm{C}-\mathrm{HUM}$ & 5,3 & 4,8 & 0,2 & 11,8 & 8,9 & 0,5 & $-0,7$ & 56,1 \\
\hline
\end{tabular}


em áreas sob manejo do solo conservacionista ou mesmo convencional (Leite et al., 2010). O aumento do tamanho deste compartimento é fundamental na recuperação de áreas degradadas já que há uma associação direta com a melhoria da qualidade do solo decorrente do aumento no fornecimento de nutrientes e estabilidade de agregados do solo. Os valores de média e mediana foram iguais dentro da mesma camada $\left(0,5 \mathrm{~g} \mathrm{~kg}^{-1}: 0,0-0,05 \mathrm{~m} ; 0,4 \mathrm{~g} \mathrm{~kg}^{-1}: 0,10-0,20 \mathrm{~m}\right)$ indicando que as medidas de tendência central são dominadas por valores típicos na distribuição, como estabelecido por Cambardella et al. (1994).

O C-FAF apresentou os menores teores no solo comparado ao C-FAH e ao C-HUM, com variações de 0,1 a 3,3 $\mathrm{g} \mathrm{kg}^{-1} \mathrm{e}$ maior média na camada de $0,0-0,05 \mathrm{~m}\left(1,0 \mathrm{~g} \mathrm{~kg}^{-1}\right)$. Valor semelhante foi reportado por Favero et al. (2008) que observaram, na mesma camada, baixos teores de FAF $\left(0,99 \mathrm{~g} \mathrm{~kg}^{-1}\right)$ em uma área degradada comparada com o sistema agroflorestal e pastagem na região do Vale do Rio Doce, Minas Gerais. Para o C-FAH, as variações nos teores, considerando-se todas as camadas, foram de 0,1 a $5,7 \mathrm{~g} \mathrm{~kg}^{-1}$ com a maior média $(2,1 \mathrm{~g}$ $\mathrm{kg}^{-1}$ ) também observada na camada 0,0-0,05 m (Tabela 1). Os teores de C-HUM oscilaram entre 0,2 e 13,5 $\mathrm{g} \mathrm{kg}^{-1} \mathrm{com}$ a maior média $\left(5,5 \mathrm{~g} \mathrm{~kg}^{-1}\right)$ verificada na camada $0,05-0,10 \mathrm{~m}$, o que representa $67 \%$ do COT. Esta predominância do C-HUM em comparação com a C-FAF e C-FAH e outras frações, tem sido observada em outros estudos (Santos et al., 2013; Guareschi et al., 2013) e está relacionada à sua insolubilidade e resistência à biodegradação favorecida pela formação de complexos argilohúmicos estáveis (Grinhut et al., 2007).

Os valores observados para carbono nas substâncias húmicas foram muito baixos (menor que 1\%), o que está associado diretamente ao processo de degradação observado na área e a na contribuição ainda insuficiente do pinhão-manso em termos de aporte de biomassa, muito provavelmente pelo pouco tempo de adoção, caso em que a inclusão de gramíneas pode favorecer a formação de agregados mais estáveis que se relacionam com o aumento do teor de $\mathrm{C}$ no solo ocasionando, assim, uma reconstrução da matéria orgânica do solo.
Comparando com outras áreas na região consideradas não degradadas, ou bem manejadas, como aquelas sob plantio direto ou integração lavoura-pecuária, observa-se que os teores de carbono são, para as mesmas profundidades, duas vezes maiores, decorrente em especial da maior entrada de resíduos e menor perda por erosão, conforme reportado por Campos et al. (2013).

Os coeficientes de assimetria e curtose para as variáveis em estudo indicam proximidade à distribuição normal; para o CL, o valor de assimetria foi - 0,1 na camada de 0,05-0,10 m; para as demais variáveis, com exceção do CL na camada de 0,0-0,05 $\mathrm{cm}$, observaram-se coeficientes de assimetria positivos com os maiores valores verificados no C-FAF (1,4: 0,0-0,05 m) e os menores no CL (0,4: 0,0-0,05 m). Quando o valor observado para este coeficiente for zero, a distribuição será simétrica; se for positivo, a distribuição será assimétrica à direita e, se for negativo é assimétrica à esquerda (Crespo, 1998) (Tabela 1).

O COT, CL e HUM apresentaram CV médio em todas as camadas variando de 32,8 a $58,9 \%$; a FAF nas camadas de 0,0-0,05 e $0,10-0,20 \mathrm{~m}$ e a FAH na camada de $0,10-0,20 \mathrm{~m}$ apresentaram CV alto (>64\%) (Tabela 1$)$.

Conforme estabelecido por Nogueira (2007), CV maior que $35 \%$ indica que a série de dados é heterogênea e a média tem pouco significado; se for maior que $65 \%$ a série é muito heterogênea e a média não tem significado algum porém se for menor que $35 \%$ a série será homogênea e a média terá significado podendo ser utilizada como representativa da série de onde foi obtida. Desta forma, observou-se que o C-FAF nas camadas de 0,0-0,05 e 0,10-0,20 m e C-FAH na camada de 0,10-0,20 m, apresentaram série de dados muito heterogêneos cuja média tem pouco significado.

Houve dependência espacial para o COT e CL nas três camadas e para C-FAF e C-FAH nas duas últimas $(0,05-0,10$ e 0,10-0,20 m). Por outro lado, apenas C-FAF e C-HUM apresentaram, na camada de 0,0-0,05 $\mathrm{m}$, efeito pepita puro, ou seja, uma distribuição aleatória na zona de estudo e independência espacial (Tabela 3). O efeito pepita puro é importante e indica variabilidade não explicada podendo

Tabela 3. Estimativa dos parâmetros dos modelos dos semivariogramas ajustados para o carbono orgânico total (COT), carbono lábil $(\mathrm{CL})$, carbono da fração ácido fúlvico (C-FAF), ácido húmico (C-FAH) e fração humina (C-HUM), nas camadas de 0-0,05 m; 0,05-0,10 m e 0,10-0,20 m de um Argissolo Vermelho-Amarelo, em Gilbués-Piauí

\begin{tabular}{|c|c|c|c|c|c|c|}
\hline Atributo & Co & $C_{1}+C_{0}$ & A & IDE & Classificação & Modelo \\
\hline \multicolumn{7}{|c|}{$0-0,05 \mathrm{~m}$} \\
\hline COT & 0,90000 & 9,93000 & 60,70 & 9 & Forte & Esférico \\
\hline $\mathrm{CL}$ & 0,00060 & 0,03200 & 52,10 & 1 & Forte & Esférico \\
\hline C-FAF & 0,55583 & 0,55583 & ----- & 100 & Efeito pepita puro & Linear \\
\hline C-FAH & 0,69200 & 2,83600 & 310,90 & 24 & Forte & Exponencial \\
\hline $\mathrm{C}-\mathrm{HUM}$ & 17,86650 & 17,86650 & ------ & 100 & Efeito pepita puro & Linear \\
\hline \multicolumn{7}{|c|}{$0,05-0,10 \mathrm{~m}$} \\
\hline COT & 1,09000 & 12,45000 & 66,50 & 8 & Forte & Esférico \\
\hline $\mathrm{CL}$ & 0,01717 & 0,04884 & 195,00 & 35 & Moderada & Exponencial \\
\hline C-FAF & 0,08380 & 0,29460 & 83,60 & 28 & Moderada & Gaussiano \\
\hline C-FAH & 0,22900 & 1,45100 & 237,90 & 15 & Forte & Esférico \\
\hline C-HUM & 0,01000 & 10,86000 & 92,00 & 0 & Forte & Esférico \\
\hline \multicolumn{7}{|c|}{$0,10-0,20 \mathrm{~m}$} \\
\hline COT & 0,30000 & 13,41000 & 70,20 & 2 & Forte & Esférico \\
\hline $\mathrm{CL}$ & 0,01430 & 0,05400 & 410,90 & 26 & Moderada & Exponencial \\
\hline C-FAF & 0,02300 & 0,19400 & 40,80 & 11 & Forte & Gaussiano \\
\hline C-FAH & 0,06700 & 0,88400 & 57,00 & 7 & Forte & Gaussiano \\
\hline C-HUM & 0,01000 & 10,27000 & 39,60 & 0 & Forte & Gaussiano \\
\hline
\end{tabular}

Efeito pepita $(\mathrm{Co})$, patamar $\left(\mathrm{C}_{1}+\mathrm{C} 0\right)$, Alcance $(\mathrm{A})$, índice de dependência espacial (IDE) 
ser decorrente de erros de medidas ou microvariações não detectadas considerando a distância de amostragem utilizada (Soares, 2006).

Observou-se, para o COT nas três camadas, dependência espacial forte, que pode ser controlada por meio da variabilidade inerente às propriedades do solo, como a textura do solo e mineralogia, como preconizado por Cambardella et al. (1994). Por outro lado, o CL nas camadas de 0,05-0,10 e 0,10-0,20 m e C-FAF na camada de 0,05-0,10 m apresentaram dependência espacial moderada ( $25 \% \leq \mathrm{IDE} \leq 75 \%)$.

O alcance, importante para a interpretação dos semivariogramas por indicar a distância até onde os pontos amostrais estão correlacionados entre si, foi diferente nas camadas de 0,0-0,05; 0,05-0,10 e 0,10-0,20 m (Tabela 2). O COT foi o atributo que apresentou menor variabilidade entre camadas com valores de alcance semelhantes $(60,70 ; 66,50$; $70,20 \mathrm{~m})$.
O maior valor de alcance foi observado no CL com 410,90 $\mathrm{m}(0,10-0,20 \mathrm{~m})$, indicando que a distribuição deste atributo é mais homogênea pois ocorre em uma área de maior raio (Tabela 2). A maior continuidade do CL na camada de 0,10 0,20 m é devida, possivelmente, à menor influência do manejo, diferente das camadas mais superficiais que são as mais susceptíveis às alterações antrópicas. Por outro lado, o C-HUM, que apresentou o menor alcance, 39,60 m (0,10-0,20 m), tem maior descontinuidade na sua distribuição espacial. Portanto, além da variabilidade vertical há, também, a variabilidade horizontal, confirmada pelos diferentes valores de alcance das variáveis em diferentes profundidades.

A maioria dos dados se ajustou ao semivariograma de modelo esférico seguido do gaussiano, exponencial e do linear (Figura 1). A seleção dos modelos foi realizada com base no melhor coeficiente de determinação $\left(\mathrm{R}^{2}\right)$. Por meio da análise visual pode-se verificar que as variáveis apresentaram
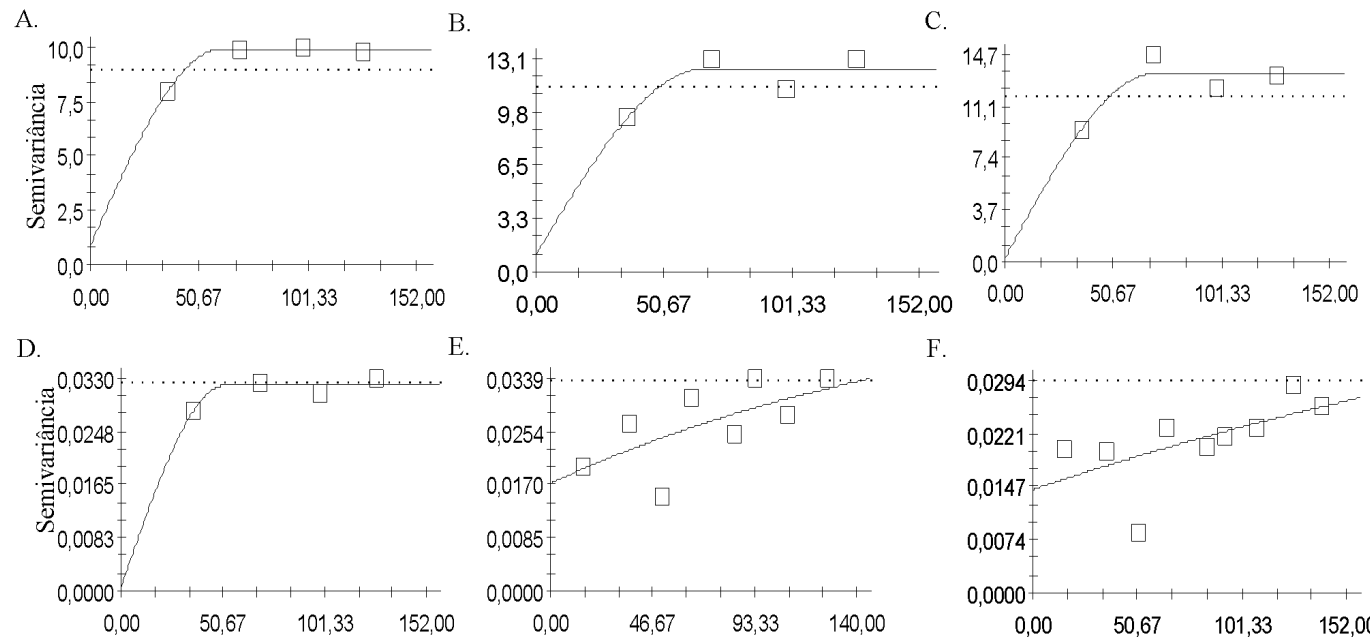

E.

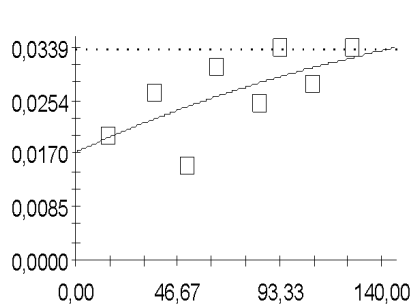

F.

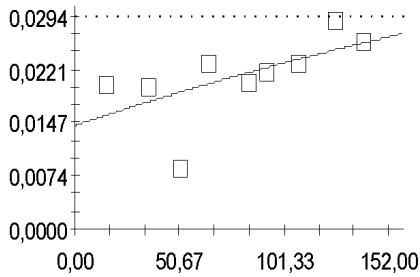

G.

H.
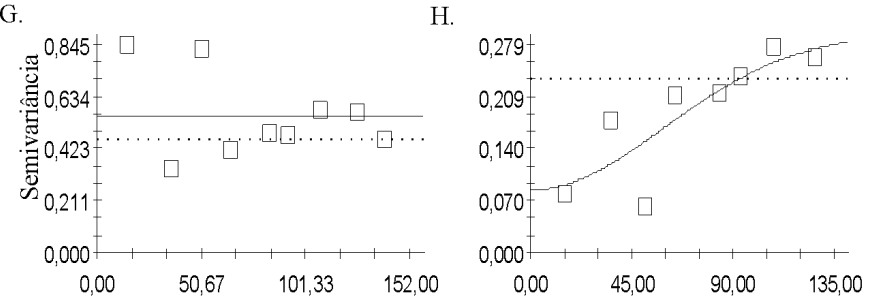

I.

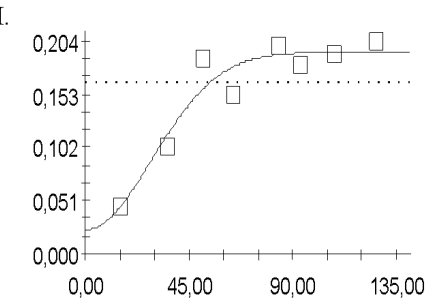

$\mathrm{J}$

$\mathrm{K}$.
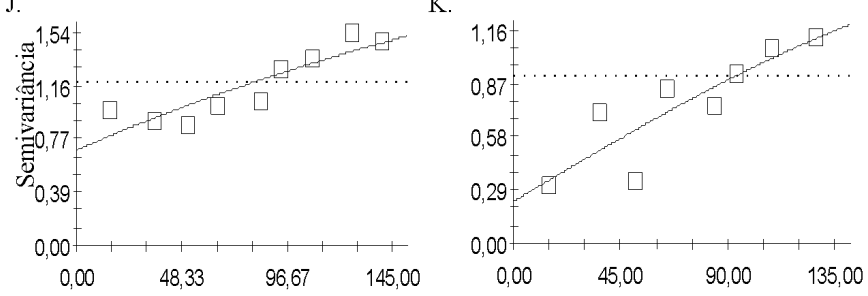

M. N.

L.

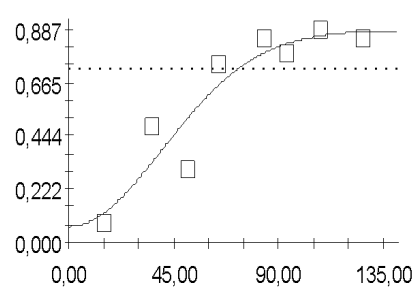

O.
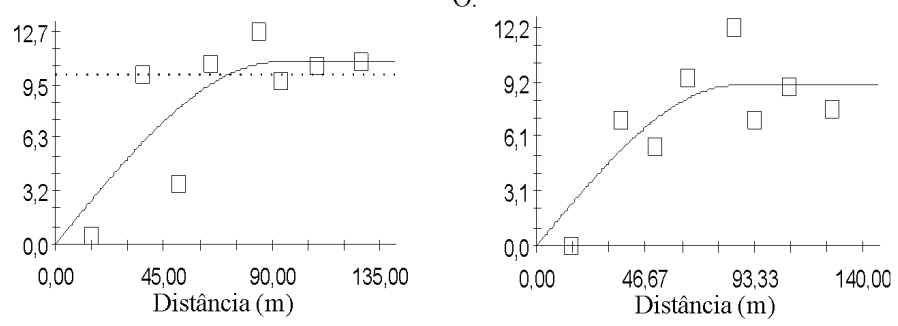

Figura 1. Modelos dos semivariogramas nas camadas de 0-0,05 m, 0,05-0,10 m e 0,10-0,20 m, respectivamente para carbono orgânico total $(A, B, C)$, carbono lábil $(D, E, F)$, carbono da fração ácido fúlvico $(C-F A F)(G, H, I)$, fração ácido húmico $(\mathrm{C}-\mathrm{FAH})(\mathrm{J}, \mathrm{K}, \mathrm{L})$ e fração humina (C-HUM) $(\mathrm{M}, \mathrm{N}, \mathrm{O})$ de um Argissolo Vermelho-Amarelo, em Gilbués-Piauí 
dependência espacial, exceto as variáveis C-FAF e C-HUM na camada de 0,0-0,05 m.

Por meio dos mapas temáticos gerados observou-se que os teores de COT e CL diminuíram com o aumento da profundidade (Figura 2). O COT foi o atributo que teve maior variação de uma profundidade para outra e maiores teores concentrados na camada superficial $(0,0-0,05 \mathrm{~m})$ com predomínio dos tons de verde e amarelo. O CL teve baixa variabilidade vertical com teores semelhantes entre as profundidades, em média $0,5 \mathrm{~g} \mathrm{~kg}^{-1}$, com maior homogeneidade
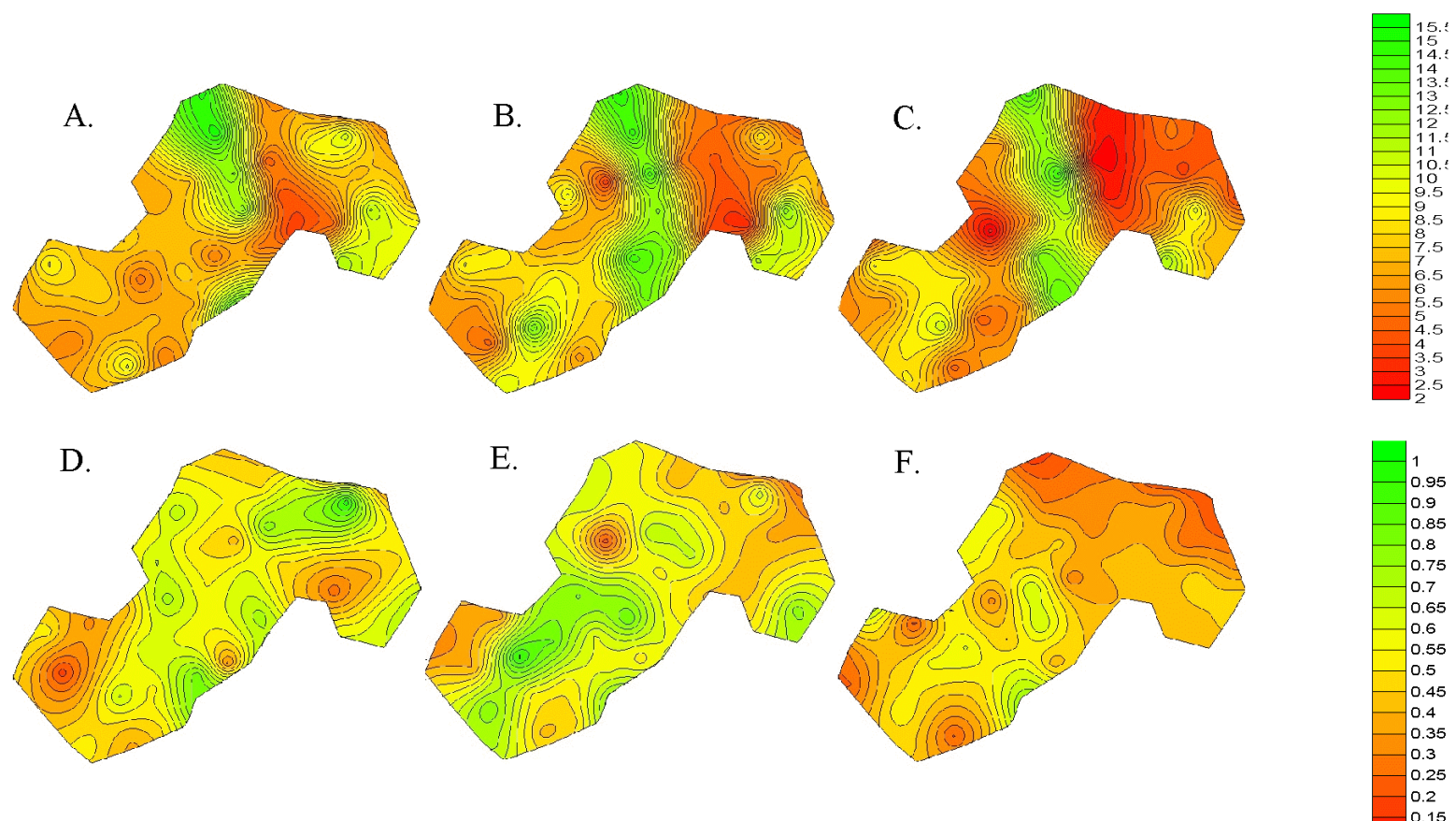

Figura 2. Mapas de distribuição espacial nas camadas de 0-0,05 m, 0,05-0,10 m e 0,10-0,20 m, respectivamente, para carbono orgânico total (COT) (A,B,C) e carbono lábil (CL) (D,E,F) de um Argissolo Vermelho-Amarelo, em Gilbués-Piauí

A.
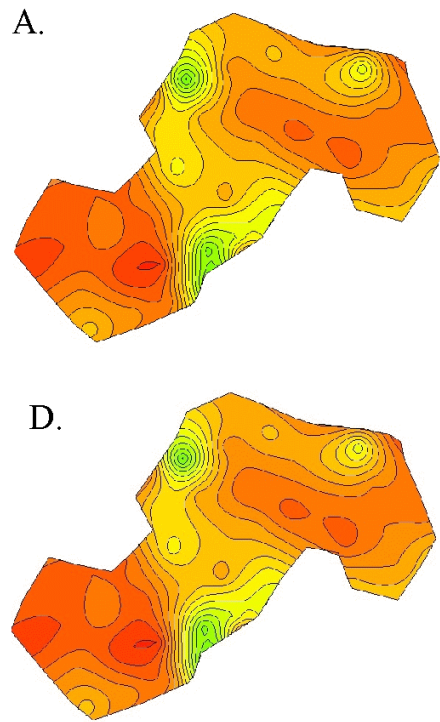

G.

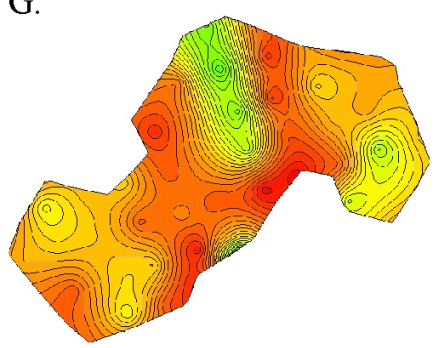

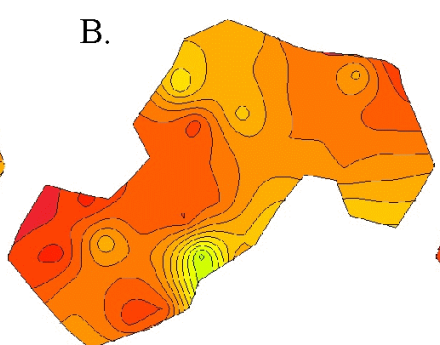
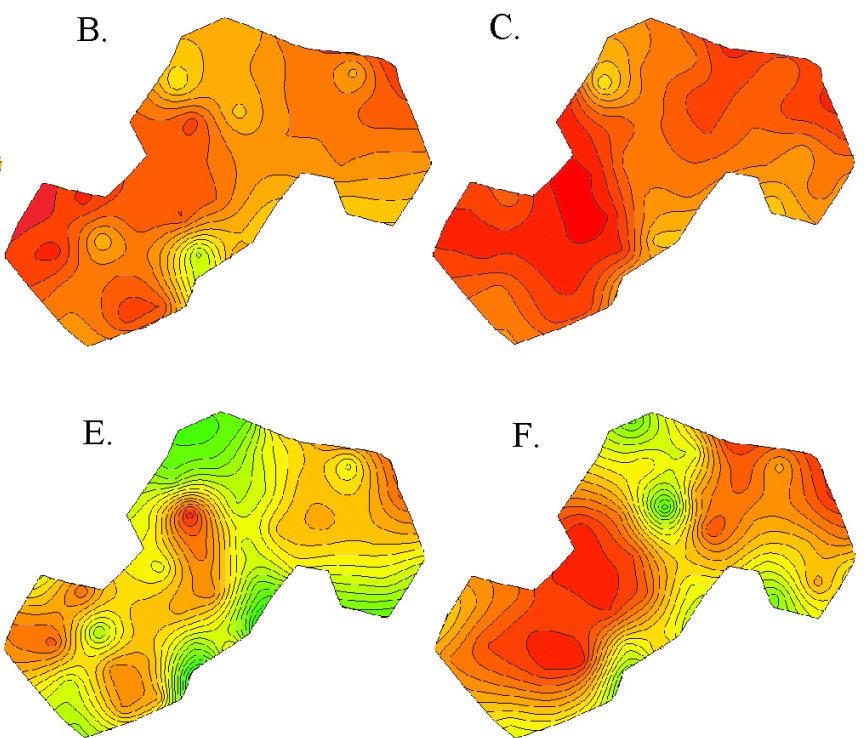

$\mathrm{H}$

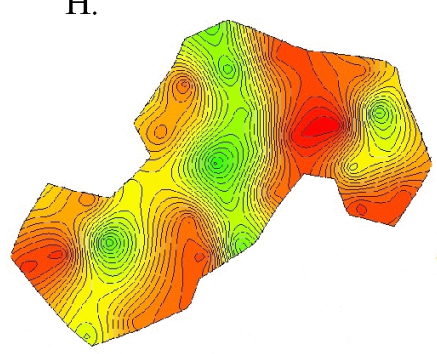

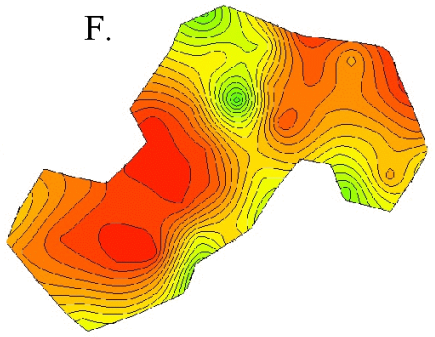

I.

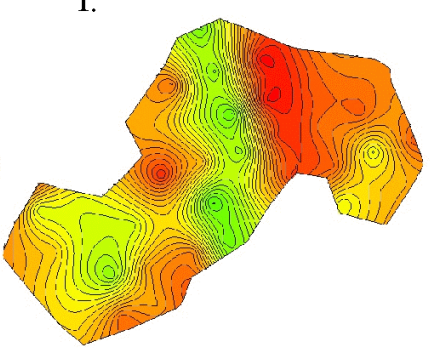

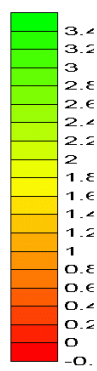
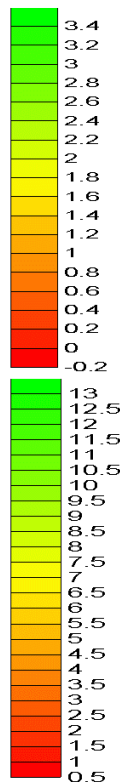

Figura 3. Mapas de distribuição espacial nas camadas de 0-0,05 m, 0,05-0,10 m e 0,10-0,20 m, respectivamente para carbono da fração ácido fúlvico $(C-F A F)(A, B, C)$, fração ácido húmico $(C-F A H)(D, E, F)$ e fração humina $(C-H U M)(G, H, I)$, de um Argissolo Vermelho-Amarelo, em Gilbués-Piauí 
na camada de 0,10-0,20 m, o que é confirmado pelo valor de alcance gerado pelo semivariograma nesta camada, que foi de 410,90 m (Figura 1F).

Nos mapas de distribuição espacial gerados para as frações estáveis (C-FAF; C-FAH; C-HUM), observou-se que não há um padrão homogêneo quanto à distribuição e assim como para as demais variáveis avaliadas, predomínio na camada de 0-0,05 m, com maiores valores máximos observados nos teores de C-HUM $\left(13,5 ; 12,8 ; 11,8 \mathrm{~g} \mathrm{~kg}^{-1}\right)$, seguida de C-FAH $\left(5,7 ; 4,5 ; 3,3 \mathrm{~g} \mathrm{~kg}^{-1}\right)$ e C-FAF $\left(3,3 ; 2,3 ; 1,6 \mathrm{~g} \mathrm{~kg}^{-1}\right)$, nas camadas de $0-0,05 ; 0,05-0,10$; e $0,10-0,20 \mathrm{~m}$, respectivamente (Figura 3). Maiores teores de huminas em comparação com as demais frações da MOS são observados em solos tropicais e atribuídos tanto à hidrofobicidade da humina como à forte interação organo-mineral (Fontana et al., 2006).

\section{Conclusões}

1. Com exceção dos C-FAF e C-HUM (0-0,05 m), os demais atributos estudados apresentaram dependência espacial classificada como moderada ou forte, com alcances variando de 39,60 a $410,90 \mathrm{~m}$.

2. A maioria dos dados se ajustou ao semivariograma de modelo esférico, seguido do gaussiano, exponencial e do linear.

3. A análise da distribuição espacial aliada à krigagem possibilitou o mapeamento da variabilidade espacial das frações lábeis e estáveis da matéria orgânica nas três profundidades proporcionando melhor visualização do seu comportamento na área de estudo.

4. Os baixos valores observados para o carbono orgânico total e suas frações, lábeis ou estáveis, realçam o estado de degradação do solo e a contribuição ainda incipiente do pinhão-manso indicando, portanto, a necessidade de aumento na entrada de carbono por meio da inclusão de espécies com elevado aporte de resíduos.

\section{Agradecimentos}

Ao CNPq, pelo auxílio financeiro.

\section{Literatura Citada}

Beheshtia, A.; Raiesia, F.; Golchin, A. Soil properties, C fractions and their dynamics in land use conversion from native forests to croplands in northern Iran. Agriculture, Ecosystems and Environment, v.148, p.121-133, 2012. http://dx.doi.org/10.1016/j. agee.2011.12.001

Blair. G. J.; Lefroy, R. D. B. E.; Lisle, L. Soil carbon fractions based on their degree of oxidation, and development of a carbon management index for agricultural systems. Australian Journal of Agricultural Research., v.46, p.1459-1466, 1995. http://dx.doi. org/10.1071/AR9951459

Brasil. Ministério do Meio Ambiente. Mapa de ocorrência de desertificação e áreas de atenção especial no Brasil. Brasília: MMA/SRH. 1998.

Cambardella, C. A.; Moorman, T. B.; Novak, J. M.; Parkin, T. B.; Karlen, D. L.; Turvo, R. F.; Konopa, A. E. Field-scale variability of soil properties in central Iowa soils. Soil Science Society of American Journal, v.58, p.1501-1511, 1994. http://dx.doi. org/10.2136/sssaj1994.03615995005800050033x
Campos, L. P.; Leite, L. F. C.; Maciel, G. A.; Brasil, E. L.; Iwata, B. F. Estoques e frações de carbono orgânico em Latossolo Amarelo submetido a diferentes sistemas de manejo. Pesquisa Agropecuária Brasileira. v. 48, p. 304-312, 2013. http://dx.doi.org/10.1590/ S0100-204X2013000300009

Chaves, L. H. G.; Farias, C. H. A. Variabilidade espacial do estoque de carbono nos Tabuleiros Costeiros da Paraíba: Solo cultivado com cana-de-açúcar. Revista Brasileira de Ciências Agrárias, v.3, p.20-25, 2008. http://dx.doi.org/10.5039/agraria.v3ila235

Crespo, A. A. Estatística fácil. 16.ed. São Paulo: Saraiva, 1998. 207p.

EMBRAPA - Empresa Brasileira de Pesquisa Agropecuária. Sistema Brasileiro de Classificação de Solos. Brasília: Embrapa Solos, 2006. 306p.

Favero, C.; Lovo, I. C.; Mendonça, E. S. Recuperação de área degradada com sistema agroflorestal no Vale do rio doce, Minas gerais. Revista Árvore, v.32, p.861-868, 2008. http://dx.doi.org/10.1590/ S0100-67622008000500011

Figueiredo, C. C.; I; Resck, D. V. S.; Carneiro, M. A. C. Labile and stable fractions of soil organic matter under management systems and native cerrado. Revista Brasileira de Ciência do Solo v.34, p.907916, 2010. http://dx.doi.org/10.1590/S0100-06832010000300032

Fontana, A.; Pereira, M. G.; Loss, A.; Cunha, T. J. F.; Salton, J. C. Atributos de fertilidade e frações húmicas de um Latossolo Vermelho no Cerrado. Pesquisa Agropecuária Brasileira, v.41, p.847-853. 2006. http://dx.doi.org/10.1590/S0100-204X2006000500018

Grinhut, T.; Hadar, Y.; Chen, Y. Degradation and transformation of humic substances by saprotrophic fungi: Processes and mechanisms. Fungal Biology Reviews, v.21, p.179-189, 2007. http://dx.doi.org/10.1016/j.fbr.2007.09.003

Guareschi, R. F.; Pereira, M. G.; Perin, A. Frações da matéria orgânica em áreas de Latossolo sob diferentes sistemas de manejo no Cerrado do estado de Goiás. Ciências Agrárias, v.34, p.2615-2628, 2013. http://dx.doi.org/10.5433/1679-0359.2013v34n6p2615

Imoke, D. E.; Ibu, J. U.; Omonya, O. C.; Nwabueze, J. O.; Njar, G. N. Effects of land degradation on soil productivity in Calabar South Local Government Area, Nigeria. European Journal of Social Sciences, v.18, p.21-34, 2010.

Kelleher, B. P.; Simpson, M. J.; Simpson, A. J. Assessing the fate and transformation of plant residues in the terrestrial environment using HR-MAS NMR spectroscopy. Geochimica et Cosmochimica Acta, v.7, p.4080-4094, 2006. http://dx.doi.org/10.1016/j.gca.2006.06.012

Kononova, M. M. Materia orgánica del suelo: Su naturaleza, propiedades y métodos de investigación. Barcelona: Oikos-Tau, 1982. 364p.

Leite, L. F. C.; Oliveira, F. C.; Araujo, A. S. F.; Galvao, S. R. S.; Lemos, J. O; Silva, E. F. L. Soil organic carbon and biological indicators in an Acrisol under tillage systems and organic management in north-eastern Brazil. Australian Journal of Soil Research, v.48, p.258-265, 2010. http://dx.doi.org/10.1071/SR09122

Matias, M. C. B.; Salviano, A. A. C.; Leite, L. F. C.; Galvão, S. R. S. Propriedades químicas em Latossolo Amarelo de Cerrado do Piauí sob diferentes sistemas de manejo. Revista Ciência Agronômica, v.40, p.356-362, 2009.

Marchetti, A.; Piccini, C.; R. Francaviglia, R.; Mabit, L. Spatial Distribution of Soil Organic Matter Using Geostatistics: A key indicator to assess soil degradation status in central Italy. Pedosphere, v.22, p.230-242, 2012. http://dx.doi.org/10.1016/S1002-0160(12)60010-1

Moebius-Clune, B. N.; Elsevierb, I. H.; Crawfordc, B. A.; Traumannd, N. M.; Schindelbecka, R. R.; Esa, H. M. van. Moving authentic soil research into high school classrooms: Student engagement and learning. Natural Sciences Education, v.40, p.102-113, 2011. http://dx.doi.org/10.4195/jnrlse.2010.0019k 
Nogueira, M. C. S. Experimentação agronômica I. Piracicaba: Universidade de São Paulo, 2007. 463p.

Sales, M. C. L. Degradação ambiental em Gilbués. Mercator - Revista de Geografia da UFC, n.4, p.115-124. 2003.

Salvo, L.; Hernandez, J.; Ernest, O. Distribution of soil organic carbon in different size fractions, under pasture and crop rotations with conventional tillage and no-till systems. Soil \& Tillage Research, v.109, p.116-122, 2010. http://dx.doi.org/10.1016/j.still.2010.05.008

Santos, D. C.; Farias, M. O.; Lima, C. L. R.; Kunde, R. J.; Pillon, C. N.; Flores, C. A. Fracionamento químico e físico da matéria orgânica de um Argissolo Vermelho sob diferentes sistemas de uso. Ciência Rural, v.43, p.838-844, 2013. http://dx.doi.org/10.1590/S010384782013005000037

Shang, C.; Tiessen, H. Organic matter lability in a tropical oxisol: Evidence from shifting cultivation, chemical oxidation, particle size, density, and magnetic fractionations. Soil Science, v.162, p.795807, 1997. http://dx.doi.org/10.1097/00010694-199711000-00004

Soares, A. Geoestatística para as ciências da terra e do ambiente. Lisboa: IST Press, 2.ed. 2006. 214p.
Stevenson, F.J. Humus chemistry: Genesis, composition, reactions. New York: Willey \& Sons Inc., 1994. 496p.

von Lützow, M.; Kogel-Knabner, I.; Wkscmitt, K.; Flessa, H.; Guggenberger, G.; Matzner, E.; Marschner, B. SOM fractionation methods: Relevance to functional pools and to stabilization mechanisms. Soil Biology Biochemistry, v.39, p.2183-2207, 2007. http://dx.doi.org/10.1016/j.soilbio.2007.03.007

Warrick, A. W.; Nielsen, D. R. Spatial variability of soil physical properties in the field. In: Hillel, D. (ed.). Applications of soil physics. New York: Academic Press, 1980. p.319-344. http:// dx.doi.org/10.1016/B978-0-12-348580-9.50018-3

Yeomans, J. C.; Bremner, J. M. A rapid and precise method for routine determination of organic carbon in soil. Communications in Soil Science and Plant Analysis, v.19, p.1467-1476, 1988. http://dx.doi. org/10.1080/00103628809368027

Zong-Ming, W.; Bai, Z.; Kai-Shan, S.; Dian-Wei, L.; Chun-Ying, R. Spatial variability of soil organic carbon under maize monoculture in the song-nen plain, Northeast China. Pedosphere, v.20, p.80-89, 2010. http://dx.doi.org/10.1016/S1002-0160(09)60285-X 